\title{
14q24.1q24.3 microdeletion syndrome
}

INSERM

\section{Source}

INSERM. (1999). Orphanet: an online rare disease and orphan drug data base.

14q24.1924.3 microdeletion syndrome. ORPHA:401935

$14 \mathrm{q} 24.1 \mathrm{q} 24.3$ microdeletion syndrome is a rare, genetic, syndromic intellectual disability characterized by mild intellectual disability, delayed speech development, congenital heart defects, brachydactyly and dysmorphic facial features. 